\title{
TRACES OF HÖRMANDER ALGEBRAS ON DISCRETE SEQUENCES
}

\author{
XAVIER MASSANEDA, JOAQUIM ORTEGA-CERDÀ, \\ AND MYRIAM OUNAÏES
}

\begin{abstract}
We show that a discrete sequence $\Lambda$ of the complex plane is the union of $n$ interpolating sequences for the Hörmander algebras $A_{p}$ if and only if the trace of $A_{p}$ on $\Lambda$ coincides with the space of functions on $\Lambda$ for which the divided differences of order $n-1$ are uniformly bounded. The analogous result holds in the unit disk for Korenblum-type algebras.
\end{abstract}

\section{Definitions and statement}

A function $p: \mathbb{C} \longrightarrow \mathbb{R}_{+}$, is called a weight if

(w1) There is a constant $K>0$ such that $p(z) \geq K \ln \left(1+|z|^{2}\right)$.

(w2) There are constants $D_{0}>0$ and $E_{0}>0$ such that whenever $|z-w| \leq 1$ then

$$
p(z) \leq D_{0} p(w)+E_{0} .
$$

Let $H(\mathbb{C})$ denote the space of all entire functions. We consider the algebra

$A_{p}=\left\{f \in H(\mathbb{C}), \quad \forall z \in \mathbb{C},|f(z)| \leq A e^{B p(z)}\right.$ for some $\left.A>0, B>0\right\}$.

Condition (w1) implies that $A_{p}$ contains the polynomials and (w2) that it is closed under differentiation.

Definition 1.1. Given a discrete subset $\Lambda \subset \mathbb{C}$ we denote by $A_{p}(\Lambda)$ the space of sequences $\omega(\Lambda)=\{\omega(\lambda)\}_{\lambda \in \Lambda}$ of complex numbers such that there are constants $A, B>0$ for which

$$
|\omega(\lambda)| \leq A e^{B p(\lambda)}, \quad \lambda \in \Lambda .
$$

Date: November 8, 2018.

1991 Mathematics Subject Classification. 30E05, 42A85.

Key words and phrases. Interpolating sequences, Divided differences.

Partially supported by the Picasso programme (Action Integrée) HF2006-0211. First and second authors supported by MEC grant MTM2005-008984-C02-02 and CIRIT grant 2005-SGR 00611. 
We say that $\Lambda$ is an interpolating sequence for $A_{p}$ when for every sequence $\omega(\Lambda) \in A_{p}(\Lambda)$ there exists $f \in A_{p}$ such that $f(\lambda)=\omega(\lambda)$, $\lambda \in \Lambda$. In terms of the restriction operator

$$
\begin{aligned}
\mathcal{R}_{\Lambda}: A_{p} & \longrightarrow A_{p}(\Lambda) \\
f & \mapsto\{f(\lambda)\}_{\lambda \in \Lambda},
\end{aligned}
$$

$\Lambda$ is interpolating when $\mathcal{R}_{\Lambda}\left(A_{p}\right)=A_{p}(\Lambda)$.

Definition 1.2. Let $\Lambda$ be a discrete sequence in $\mathbb{C}$ and $\omega$ a function given on $\Lambda$. The divided differences of $\omega$ are defined by induction as follows

$$
\begin{aligned}
\Delta^{0} \omega\left(\lambda_{1}\right) & =\omega\left(\lambda_{1}\right) \\
\Delta^{j} \omega\left(\lambda_{1}, \ldots, \lambda_{j+1}\right) & =\frac{\Delta^{j-1} \omega\left(\lambda_{2}, \ldots, \lambda_{j+1}\right)-\Delta^{j-1} \omega\left(\lambda_{1}, \ldots, \lambda_{j}\right)}{\lambda_{j+1}-\lambda_{1}} \quad j \geq 1 .
\end{aligned}
$$

For any $n \in \mathbb{N}$, denote

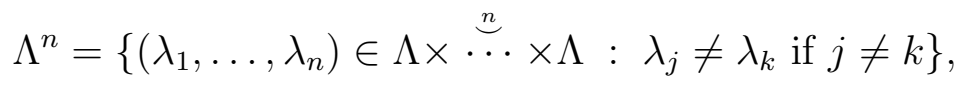

and consider the set $X_{p}^{n-1}(\Lambda)$ consisting of the functions in $\omega(\Lambda)$ with divided differences of order $n$ uniformly bounded with respect to the weight $p$, i.e., such that for some $B>0$

$$
\sup _{\left(\lambda_{1}, \ldots, \lambda_{n}\right) \in \Lambda^{n}}\left|\Delta^{n-1} \omega\left(\lambda_{1}, \ldots, \lambda_{n}\right)\right| e^{-B\left[p\left(\lambda_{1}\right)+\cdots+p\left(\lambda_{n}\right)\right]}<+\infty .
$$

Remark 1.3. It is clear that $X_{p}^{n}(\Lambda) \subset X_{p}^{n-1}(\Lambda) \subset \cdots \subset X_{p}^{0}(\Lambda)=$ $A_{p}(\Lambda)$.

To see this assume that $\omega(\Lambda) \in X_{p}^{n}(\Lambda)$, i.e., there exists $B>0$ such that

$$
\begin{array}{r}
C:=\sup _{\left(\lambda_{1}, \ldots, \lambda_{n+1}\right) \in \Lambda^{n+1}}\left|\frac{\Delta^{n-1} \omega\left(\lambda_{2}, \ldots, \lambda_{n+1}\right)-\Delta^{n-1} \omega\left(\lambda_{1}, \ldots, \lambda_{n}\right)}{\lambda_{n+1}-\lambda_{1}}\right| \times \\
\times e^{-B\left[p\left(\lambda_{1}\right)+\cdots+p\left(\lambda_{n+1}\right)\right]}<\infty .
\end{array}
$$

Then, given $\left(\lambda_{1}, \ldots, \lambda_{n}\right) \in \Lambda^{n}$ and taking $\lambda_{1}^{0}, \ldots, \lambda_{n}^{0}$ from a finite set (for instance the $n$ first $\lambda_{j}^{0} \in \Lambda$ different of all $\lambda_{j}$ ) we have

$$
\begin{aligned}
& \Delta^{n-1} \omega\left(\lambda_{1}, \ldots, \lambda_{n}\right)=\frac{\Delta^{n-1} \omega\left(\lambda_{1}, \ldots, \lambda_{n}\right)-\Delta^{n-1} \omega\left(\lambda_{1}^{0}, \lambda_{1}, \ldots, \lambda_{n-1}\right)}{\lambda_{n}-\lambda_{1}^{0}}\left(\lambda_{n}-\lambda_{0}^{1}\right)+ \\
&+ \frac{\Delta^{n-1} \omega\left(\lambda_{1}^{0}, \lambda_{1}, \ldots, \lambda_{n-1}\right)-\Delta^{n-1} \omega\left(\lambda_{2}^{0}, \lambda_{1}^{0}, \ldots, \lambda_{n-2}\right)}{\lambda_{n-1}-\lambda_{2}^{0}}\left(\lambda_{n-1}-\lambda_{2}^{0}\right)+\cdots+ \\
& \frac{\Delta^{n-1} \omega\left(\lambda_{n-1}^{0}, \ldots, \lambda_{1}^{0}, \lambda_{1}\right)-\Delta^{n-1} \omega\left(\lambda_{n}^{0}, \ldots, \lambda_{1}^{0}\right)}{\lambda_{1}-\lambda_{n}^{0}}\left(\lambda_{1}-\lambda_{n}^{0}\right)+\Delta^{n-1} \omega\left(\lambda_{n}^{0}, \ldots, \lambda_{1}^{0}\right)
\end{aligned}
$$


Then a direct estimate and (w1) show that for some $B>0$ there is a constant $K\left(\lambda_{1}^{0}, \ldots, \lambda_{n}^{0}\right)$ such that

$$
\begin{aligned}
\left|\Delta^{n-1} \omega\left(\lambda_{1}, \ldots, \lambda_{n}\right)\right| & \leq C\left(e^{B\left[p\left(\lambda_{1}^{0}\right)+\cdots+p\left(\lambda_{n}\right)\right]}+\cdots+e^{B\left[p\left(\lambda_{n-1}^{0}\right)+\cdots+p\left(\lambda_{1}\right)\right]}\right) \\
& \leq K\left(\lambda_{1}^{0}, \ldots, \lambda_{n}^{0}\right) e^{B\left[p\left(\lambda_{1}\right)+\cdots+p\left(\lambda_{n}\right)\right]},
\end{aligned}
$$

and the statement follows.

The main result of this note is modelled after Vasyunin's description of the sequences $\Lambda$ in the unit disk such that the trace of the algebra of bounded holomorphic functions $H^{\infty}$ on $\Lambda$ equals the space of (hyperbolic) divided differences of order $n$ (see [7], [8]). The analogue in our context is the following.

Theorem 1.4 (Main Theorem). The identity $\mathcal{R}_{\Lambda}\left(A_{p}\right)=X_{p}^{n-1}(\Lambda)$ holds if and only if $\Lambda$ is the union of $n$ interpolating sequences for $A_{p}$.

For the most usual of these weights there exists a complete description of the $A_{p}$-interpolating sequences, both in analytic and geometric terms. This is the case for doubling and radial weights (see [2, Corollary $4.8]$ ), or for non-isotropic weights of the form $p(z)=|\operatorname{Im} z|+\log (1+|z|)$ (see [5, Theorem 1]).

With similar techiques it should be possible to extend this result to an Hermite-type interpolation problem with multiplicites, along the lines of [6].

\section{General properties}

We begin by showing that one of the inclusions of Theorem 1.4 is inmediate.

Proposition 2.1. For all $n \in \mathbb{N}$, the inclusion $\mathcal{R}_{\Lambda}\left(A_{p}\right) \subset X_{p}^{n-1}(\Lambda)$ holds.

Proof. Let $f \in A_{p}$. Let us show by induction on $j \geq 1$ that, for certain constants $A, B>0$

$$
\left|\Delta^{j-1} f\left(z_{1}, \ldots, z_{j}\right)\right| \leq A e^{B\left[p\left(z_{1}\right)+\cdots+p\left(z_{j}\right)\right]} \quad \text { for all }\left(z_{1}, \ldots, z_{j}\right) \in \mathbb{C}^{j} .
$$

As $f \in A_{p}$, we have $\left|\Delta^{0} f\left(z_{1}\right)\right|=\left|f\left(z_{1}\right)\right| \leq A e^{B p\left(z_{1}\right)}$.

Assume that the property is true for $j$ and let $\left(z_{1}, \ldots, z_{j+1}\right) \in \mathbb{C}^{j+1}$. Fix $z_{1}, \ldots, z_{j}$ and consider $z_{j+1}$ as the variable in the function

$$
\Delta^{j} f\left(z_{1}, \ldots, z_{j+1}\right)=\frac{\Delta^{j-1} f\left(z_{2}, \ldots, z_{j+1}\right)-\Delta^{j-1} f\left(z_{1}, \ldots, z_{j}\right)}{z_{j+1}-z_{1}} .
$$


By the induction hypothesis,

$$
\begin{aligned}
& \left|\Delta^{j-1} f\left(z_{2}, \ldots, z_{j+1}\right)-\Delta^{j-1} f\left(z_{1}, \ldots, z_{j}\right)\right| \leq \\
& \quad \leq A\left(e^{B\left[p\left(z_{2}\right)+\cdots+p\left(z_{j+1}\right)\right]}+e^{B\left[p\left(z_{1}\right)+\cdots+p\left(z_{j}\right)\right]}\right) \leq 2 A e^{B\left[p\left(z_{1}\right)+\cdots+p\left(z_{j+1}\right)\right]} .
\end{aligned}
$$

Thus, if $\left|z_{j+1}-z_{1}\right| \geq 1$, we easily deduce the desired estimate. For $\left|z_{j+1}-z_{1}\right| \leq 1$, by the maximum principle and (w2):

$$
\begin{aligned}
\left|\Delta^{j} f\left(z_{1}, \ldots, z_{j+1}\right)\right| & \leq 2 A \sup _{\left|\xi-z_{1}\right|=1} e^{B\left[p\left(z_{1}\right)+\cdots+p\left(z_{j}\right)+p(\xi)\right]} \\
& \leq A e^{\left(B+D_{0}\right)\left[p\left(z_{1}\right)+\cdots+p\left(z_{j}\right)+p\left(z_{j+1}\right)\right]}
\end{aligned}
$$

Definition 2.2. A sequence $\Lambda$ is weakly separated if there exist constants $\varepsilon>0$ and $C>0$ such that the disks $D\left(\lambda, \varepsilon e^{-C p(\lambda)}\right), \lambda \in \Lambda$, are pairwise disjoint.

Remark 2.3. If $\Lambda$ is weakly separated then $X_{p}^{0}(V)=X_{p}^{n}(V)$, for all $n \in \mathbb{N}$.

To see this it is enough to prove (by induction) that $X_{p}^{0}(\Lambda) \subset X_{p}^{n}(\Lambda)$ for all $n \in \mathbb{N}$. For $n=0$ this is trivial. Assume now that $X_{p}^{0}(\Lambda) \subset$ $X_{p}^{n-1}(\Lambda)$. Given $\omega(\Lambda) \in X_{p}^{0}(\Lambda)$ we have

$$
\begin{aligned}
\left|\Delta^{n} \omega\left(\lambda_{1}, \ldots, \lambda_{n+1}\right)\right| & =\left|\frac{\Delta^{n-1}\left(\lambda_{2}, \ldots, \lambda_{n+1}\right)-\Delta^{n-1}\left(\lambda_{1}, \ldots, \lambda_{n}\right)}{\lambda_{n+1}-\lambda_{1}}\right| \\
& \leq \frac{2 A}{\varepsilon} e^{(B+C)\left[p\left(\lambda_{1}\right)+\cdots+p\left(\lambda_{n+1}\right)\right]} .
\end{aligned}
$$

Lemma 2.4. Let $n \geq 1$. The following assertions are equivalent:

(a) $\Lambda$ is the union of $n$ weakly separated sequences,

(b) There exist constants $\varepsilon>0$ and $C>0$ such that

$$
\sup _{\lambda \in \Lambda} \#\left[\Lambda \cap D\left(\lambda, \varepsilon e^{-C p(\lambda)}\right)\right] \leq n .
$$

(c) $X_{p}^{n-1}(\Lambda)=X_{p}^{n}(\Lambda)$.

Proof. (a) $\Rightarrow(\mathrm{b})$. This is clear, by the weak separation.

(b) $\Rightarrow($ a). We proceed by induction on $j=1, \ldots, n$. For $j=1$, it is again clear by the definition of weak separation. Assume the property true for $j-1$. Let $1 \geq \varepsilon>0$ and $C>0$ be such that $\sup _{\lambda \in \Lambda} \#\left[\Lambda \cap D\left(\lambda, \varepsilon e^{-C p(\lambda)}\right)\right] \leq j$. Put $\varepsilon^{\prime}=e^{-E_{0} C} \varepsilon / 2$ and $C^{\prime}=D_{0} C$. By Zorn's Lemma, there is a maximal subsequence $\Lambda_{1} \subset \Lambda$ such that the disks $D\left(\lambda, \varepsilon^{\prime} e^{-C^{\prime} p(\lambda)}\right), \lambda \in \Lambda_{1}$, are pairwise disjoint. In particular 
$\Lambda_{1}$ is weakly separated. For any $\alpha \in \Lambda \backslash \Lambda_{1}$, there exists $\lambda \in \Lambda_{1}$ such that

$$
D\left(\lambda, \varepsilon^{\prime} e^{-C^{\prime} p(\lambda)}\right) \cap D\left(\alpha, \varepsilon^{\prime} e^{-C^{\prime} p(\alpha)}\right) \neq \emptyset,
$$

otherwise $\Lambda_{1}$ would not be maximal. Then $\lambda \in D\left(\alpha, \varepsilon e^{-C p(\alpha)}\right)$, since

$$
|\lambda-\alpha|<\varepsilon^{\prime} e^{-C^{\prime} p(\lambda)}+\varepsilon^{\prime} e^{-C^{\prime} p(\alpha)}<\varepsilon e^{-C p(\alpha)},
$$

by (w2). Thus $D\left(\alpha, \varepsilon e^{-C p(\alpha)}\right)$ contains at most $j-1$ points of $\Lambda \backslash \Lambda_{1}$. We use the induction hypothesis to conclude that $\Lambda \backslash \Lambda_{1}$ is the union of $j-1$ weakly separated sequences and, by consequence, $\Lambda$ is the union of $j$ weakly separated sequences.

(b) $\Rightarrow(\mathrm{c})$. It remains to see that $X_{p}^{n-1}(\Lambda) \subset X_{p}^{n}(\Lambda)$. Given $\omega(\Lambda) \in$ $X_{p}^{n-1}(\Lambda)$ and points $\left(\lambda_{1}, \ldots, \lambda_{n+1}\right) \in \Lambda^{n+1}$, we have to estimate $\Delta^{n} \omega\left(\lambda_{1}, \ldots, \lambda_{n+1}\right)$. Under the assumption (b), at least one of these $n+1$ points is not in the disk $D\left(\lambda_{1}, \varepsilon e^{-C p\left(\lambda_{1}\right)}\right)$. Note that $\Lambda^{n}$ is invariant by permutation of the $n+1$ points, thus we may assume that $\left|\lambda_{1}-\lambda_{n+1}\right| \geq \varepsilon e^{-C p\left(\lambda_{1}\right)}$. Using the fact that $\omega(\Lambda) \in X_{p}^{n-1}(\Lambda)$, there are constants $A, B>0$ such that

$$
\begin{aligned}
\left|\Delta^{n} \omega\left(\lambda_{1}, \ldots, \lambda_{n+1}\right)\right| & \leq \frac{\left|\Delta^{n-1} \omega\left(\lambda_{2}, \ldots, \lambda_{n+1}\right)\right|+\left|\Delta^{n-1} \omega\left(\lambda_{1}, \ldots, \lambda_{n}\right)\right|}{\left|\lambda_{1}-\lambda_{n+1}\right|} \\
& \leq A e^{B\left[p\left(\lambda_{1}\right)+\cdots+p\left(\lambda_{n+1}\right)\right]}
\end{aligned}
$$

$(\mathrm{c}) \Rightarrow(\mathrm{b})$. We prove this by contraposition. Assume that for all $C, \varepsilon>0$, there exists $\lambda \in \Lambda$ such that $\#\left[\Lambda \cap D\left(\lambda, \varepsilon e^{-C p(\lambda)}\right)\right]>n$. Since $\Lambda$ has no accumulation points, for any fixed $C>0$, we can extract from $\Lambda$ a weakly separated subsequence $\mathcal{L}=\left\{\alpha^{l}\right\}_{l \in \mathbb{N}}$ such that $\#\left[(\Lambda \backslash \mathcal{L}) \cap D\left(\alpha^{l}, 1 / l e^{-C p\left(\alpha^{l}\right)}\right)\right] \geq n$ for all $l$. Let us call $\lambda_{1}^{l}, \ldots, \lambda_{n}^{l}$ the points of $\Lambda \backslash \mathcal{L}$ closest to $\alpha^{l}$, arranged by increasing distance. In order to construct a sequence $\omega(\Lambda) \in X_{p}^{n-1}(\Lambda) \backslash X_{p}^{n}(\Lambda)$, put

$$
\begin{aligned}
\omega\left(\alpha^{l}\right) & =\prod_{j=1}^{n-1}\left(\alpha^{l}-\lambda_{j}^{l}\right), \text { for all } \alpha^{l} \in \mathcal{L} \\
\omega(\lambda) & =0 \text { if } \lambda \in \Lambda \backslash \mathcal{L} .
\end{aligned}
$$

To see that $\omega(\Lambda) \in X^{n-1}(\Lambda)$ let us estimate $\Delta^{n-1} \omega\left(\lambda_{1}, \ldots, \lambda_{n}\right)$ for any given vector $\left(\lambda_{1}, \ldots, \lambda_{n}\right) \in \Lambda^{n}$. We don't need to consider the case where the points are distant, thus, as $\mathcal{L}$ is weakly separated, we may assume that at most one of the points is in $\mathcal{L}$. On the other hand, it is clear that $\Delta^{n-1} \omega\left(\lambda_{1}, \ldots, \lambda_{n}\right)=0$ if all the points are in $\Lambda \backslash \mathcal{L}$. Then, taking into account that $\Delta^{n-1}$ is invariant by permutation, we will only consider the case where $\lambda_{n}$ is some $\alpha^{l} \in \mathcal{L}$ and $\lambda_{1}, \ldots, \lambda_{n-1}$ 
are in $\Lambda \backslash \mathcal{L}$. In that case,

$$
\left|\Delta^{n-1} \omega\left(\lambda_{1}, \ldots, \lambda_{n-1}, \alpha^{l}\right)\right|=\left|\omega\left(\alpha^{l}\right)\right| \prod_{k=1}^{n-1}\left|\alpha^{l}-\lambda_{k}^{l}\right|^{-1} \leq 1
$$

as desired.

On the other hand, a similar computation yields

$$
\left|\Delta^{n} \omega\left(\lambda_{1}^{l}, \ldots, \lambda_{n}^{l}, \alpha^{l}\right)\right|=\left|\omega\left(\alpha^{l}\right)\right| \prod_{k=1}^{n}\left|\alpha^{l}-\lambda_{k}^{l}\right|^{-1}=\left|\alpha^{l}-\lambda_{n}^{l}\right|^{-1} \geq l e^{C p\left(\alpha^{l}\right)}
$$

Using (w2), for any constant $B>0$, and choosing $C=B\left(n D_{0}+1\right)$, we have

$$
\left|\Delta^{n} \omega\left(\lambda_{1}^{l}, \ldots, \lambda_{n}^{l}, \alpha^{l}\right)\right| e^{-B\left(p\left(\lambda_{1}^{l}\right)+\cdots+p\left(\lambda_{n}^{l}\right)+p\left(\alpha^{l}\right)\right)} \geq l e^{-B n E_{0}} \rightarrow+\infty .
$$

We finally conclude that $\omega(\Lambda) \notin X_{p}^{n}(\Lambda)$.

Corollary 2.5. If $\Lambda$ is an interpolating sequence, then it is weakly separated.

Proof. If $\Lambda$ is an interpolating sequence, then $\mathcal{R}_{\Lambda}\left(A_{p}\right)=X_{p}^{0}(\Lambda)$. On the other hand, by Proposition 2.1, $\mathcal{R}_{\Lambda}\left(A_{p}\right) \subset X_{p}^{1}(\Lambda)$. Thus $X_{p}^{0}(\Lambda)=$ $X_{p}^{1}(\Lambda)$. We conclude by the preceding lemma applied to the particular case $n=1$.

Lemma 2.6. Let $\Lambda_{1}, \ldots, \Lambda_{n}$ be weakly separated sequences. There exist positive constants $a, b, B_{1}, B_{2}$ and $\varepsilon>0$, a subsequence $\mathcal{L} \subset \Lambda_{1} \cup \cdots \cup \Lambda_{n}$ and disks $D_{\lambda}=D\left(\lambda, r_{\lambda}\right), \lambda \in \mathcal{L}$, such that

(i) $\Lambda_{1} \cup \cdots \cup \Lambda_{n} \subset \cup_{\lambda \in \mathcal{L}} D_{\lambda}$

(ii) $a \varepsilon e^{-B_{1} p(\lambda)} \leq r_{\lambda} \leq b \varepsilon e^{-B_{2} p(\lambda)}$ for all $\lambda \in \mathcal{L}$

(iii) $\operatorname{dist}\left(D_{\lambda}, D_{\lambda^{\prime}}\right) \geq a \varepsilon e^{-B_{1} p(\lambda)}$ for all $\lambda, \lambda^{\prime} \in \mathcal{L}, \lambda \neq \lambda^{\prime}$.

(iv) $\#\left(\Lambda_{j} \cap D_{\lambda}\right) \leq 1$ for all $j=1, \ldots, n$ and $\lambda \in \mathcal{L}$.

Proof. Let $0<\varepsilon<1$ and $C>0$ be constants such that

$\left|\lambda-\lambda^{\prime}\right| \geq \varepsilon e^{-C / D_{0}\left(p(\lambda)-E_{0}\right)}, \quad \forall \lambda, \lambda^{\prime} \in \Lambda_{j}, \lambda \neq \lambda^{\prime}, \quad \forall j=1, \ldots, n$,

where $D_{0} \geq 1$ and $E_{0} \geq 0$ are given by (w2).

We will proceed by induction on $k=1, \ldots, n$ to show the existence of a subsequence $\mathcal{L}_{k} \subset \Lambda_{1} \cup \cdots \cup \Lambda_{k}$ and constants $C_{k} \geq C, B_{k} \geq 0$ 
such that:

$(i)_{k} \quad \Lambda_{1} \cup \cdots \cup \Lambda_{k} \subset \cup_{\lambda \in \mathcal{L}_{k}} D\left(\lambda, R_{\lambda}^{k}\right)$,

$(i i)_{k} \quad 2^{-3 k} e^{-C_{k} p(\lambda)-B_{k}} \varepsilon \leq R_{\lambda}^{k} \leq \varepsilon e^{-C p(\lambda)} \sum_{j=0}^{k-1} 2^{-(3 j+2)} \leq 2 / 7 e^{-C p(\lambda)} \varepsilon$,

$(\text { iii })_{k} \quad \operatorname{dist}\left(D\left(\lambda, R_{\lambda}^{k}\right), D\left(\lambda^{\prime}, R_{\lambda^{\prime}}^{k}\right)\right) \geq 2^{-3 k} \varepsilon e^{-C_{k} p(\lambda)-B_{k}}$ for any $\lambda, \lambda^{\prime} \in \mathcal{L}_{k}, \lambda \neq \lambda^{\prime}$.

The constants $C_{k}$ and $B_{k}$ are chosen, in view of (w2), so that $C_{k} p(\lambda)+$ $B_{k} \leq C_{k+1} p\left(\lambda^{\prime}\right)+B_{k+1}$ whenever $\left|\lambda-\lambda^{\prime}\right| \leq 1$.

Then it suffices to chose $\mathcal{L}=\mathcal{L}_{n}, r_{\lambda}=R_{\lambda}^{n}, a=e^{-B_{n}} 2^{-3 n}, b=2 / 7$, $B_{1}=C_{n}$ and $B_{2}=C$. As $r_{\lambda}<e^{-C p(\lambda)} \varepsilon$, it is clear that $D\left(\lambda, r_{\lambda}\right)$ contains at most one point of each $\Lambda_{j}$, hence the lemma follows.

For $k=1$, the property is clearly verified with $\mathcal{L}_{1}=\Lambda_{1}$ and $R_{\lambda}^{1}=$ $e^{-C p(\lambda)} \varepsilon / 4$.

Assume the property true for $k$ and split $\mathcal{L}_{k}=\mathcal{M}_{1} \cup \mathcal{M}_{2}$ and $\Lambda_{k+1}=$ $\mathcal{N}_{1} \cup \mathcal{N}_{2}$, where

$$
\begin{aligned}
\mathcal{M}_{1} & =\left\{\lambda \in \mathcal{L}_{k}: D\left(\lambda, R_{\lambda}^{k}+2^{-3 k-2} \varepsilon e^{-C_{k} p(\lambda)-B_{k}}\right) \cap \Lambda_{k+1} \neq \emptyset\right\}, \\
\mathcal{N}_{1} & =\Lambda_{k+1} \cap \bigcup_{\lambda \in \mathcal{L}_{k}} D\left(\lambda, R_{\lambda}^{k}+2^{-3 k-2} \varepsilon e^{-C_{k} p(\lambda)-B_{k}}\right), \\
\mathcal{M}_{2} & =\mathcal{L}_{k} \backslash \mathcal{M}_{1} \\
\mathcal{N}_{2} & =\Lambda_{k+1} \backslash \mathcal{N}_{1} .
\end{aligned}
$$

Now, we put $\mathcal{L}_{k+1}=\mathcal{L}_{k} \cup \mathcal{N}_{2}$ and define the radii $R_{\lambda}^{k+1}$ as follows:

$$
R_{\lambda}^{k+1}= \begin{cases}R_{\lambda}^{k}+2^{-3 k-2} \varepsilon e^{-C_{k} p(\lambda)-B_{k}} & \text { if } \lambda \in \mathcal{M}_{1}, \\ R_{\lambda}^{k} & \text { if } \lambda \in \mathcal{M}_{2}, \\ 2^{-3 k-3} \varepsilon e^{-C_{k+1} p(\lambda)-B_{k+1}} & \text { if } \lambda \in \mathcal{N}_{2} .\end{cases}
$$

It is clear that

$$
\Lambda_{1} \cup \cdots \cup \Lambda_{k+1} \subset \bigcup_{\lambda \in \mathcal{L}_{k+1}} D\left(\lambda, R_{\lambda}^{k+1}\right)
$$

and, by the induction hypothesis,

$$
2^{-3 k-3} \varepsilon e^{\left.-C_{k+1} p(\lambda)+B_{k+1}\right)} \leq R_{\lambda}^{k+1} \leq \varepsilon e^{-C p(\lambda)} \sum_{j=0}^{k} 2^{-3 j-2} \leq 2 / 7 \varepsilon e^{-C p(\lambda)} .
$$

In order to prove $(i i i)_{k}$ take now $\lambda, \lambda^{\prime} \in \mathcal{L}_{k+1}, \lambda \neq \lambda^{\prime}$. We will verify that

$\operatorname{dist}\left(D\left(\lambda, R_{\lambda}^{k+1}\right), D\left(\lambda^{\prime}, R_{\lambda^{\prime}}^{k+1}\right)\right)=\left|\lambda-\lambda^{\prime}\right|-R_{\lambda}^{k+1}-R_{\lambda^{\prime}}^{k+1} \geq 2^{-3 k-3} \varepsilon e^{-C_{k+1} p(\lambda)-B_{k+1}}$

by considering different cases. 
If $\lambda, \lambda^{\prime} \in \mathcal{L}_{k}$ and $p(\lambda) \leq p\left(\lambda^{\prime}\right)$, then

$$
\begin{aligned}
\operatorname{dist}\left(D\left(\lambda, R_{\lambda}^{k+1}\right), D\left(\lambda^{\prime}, R_{\lambda^{\prime}}^{k+1}\right)\right) & \geq\left|\lambda-\lambda^{\prime}\right|-R_{\lambda}^{k}-R_{\lambda^{\prime}}^{k}-2^{-3 k-1} \varepsilon e^{-C_{k} p(\lambda)-B_{k}} \\
& \geq 2^{-3 k-1} \varepsilon e^{-C_{k} p(\lambda)-B_{k}} .
\end{aligned}
$$

Assume now $\lambda, \lambda^{\prime} \in \mathcal{N}_{2}$ and $p(\lambda) \leq p\left(\lambda^{\prime}\right)$. Condition (2.1) implies $\left|\lambda-\lambda^{\prime}\right| \geq \varepsilon e^{-C p(\lambda)}$, hence

$$
\operatorname{dist}\left(D\left(\lambda, R_{\lambda}^{k+1}\right), D\left(\lambda^{\prime}, R_{\lambda^{\prime}}^{k+1}\right)\right) \geq\left(1-2^{-3 k-2}\right) \varepsilon e^{-C p(\lambda)} .
$$

If $\lambda \in \mathcal{M}_{1}$ and $\lambda^{\prime} \in \mathcal{N}_{2}$ there exists $\beta \in \mathcal{N}_{1}$ such that $|\lambda-\beta| \leq R_{\lambda}^{k+1}$. There is no restriction in assuming that $\left|\lambda-\lambda^{\prime}\right| \leq 1$. Then, using (2.1) on $\beta, \lambda^{\prime} \in \Lambda_{k+1}$, we have

$$
\left|\lambda-\lambda^{\prime}\right| \geq\left|\beta-\lambda^{\prime}\right|-|\lambda-\beta| \geq \varepsilon e^{-C / D_{0}\left(p(\beta)-E_{0}\right)}-R_{\lambda}^{k+1} \geq \varepsilon e^{-C p(\lambda)}-R_{\lambda}^{k+1} .
$$

The definition of $R_{\lambda^{\prime}}^{k+1}$ together with the estimate $R_{\lambda}^{k+1} \leq 2 / 7 \varepsilon e^{-C p(\lambda)}$ yield

$$
\begin{aligned}
& \operatorname{dist}\left(D\left(\lambda, R_{\lambda}^{k+1}\right), D\left(\lambda^{\prime}, R_{\lambda^{\prime}}^{k+1}\right)\right) \geq \varepsilon e^{-C p(\lambda)}-2 R_{\lambda}^{k+1}-R_{\lambda^{\prime}}^{k+1} \\
& \geq \varepsilon e^{-C p(\lambda)}-2 R_{\lambda}^{k}-2^{-3 k-1} \varepsilon e^{-C_{k} p(\lambda)-B_{k}}-2^{-3 k-3} \varepsilon e^{-C_{k+1} p\left(\lambda^{\prime}\right)-B_{k+1}} \\
& \geq \varepsilon e^{-C p(\lambda)}-\frac{4}{7} \varepsilon e^{-C p(\lambda)}-2^{-3 k} \varepsilon e^{-C_{k} p(\lambda)-B_{k}} \geq \varepsilon e^{-C p(\lambda)}\left(3 / 4-2^{-3 k}\right),
\end{aligned}
$$

as required.

Finally, if $\lambda \in \mathcal{M}_{2}$ and $\lambda^{\prime} \in \mathcal{N}_{2}$, again, assuming that $\left|\lambda-\lambda^{\prime}\right| \leq 1$, we have

$$
\begin{aligned}
\operatorname{dist}\left(D\left(\lambda, R_{\lambda}^{k+1}\right), D\left(\lambda^{\prime}, R_{\lambda^{\prime}}^{k+1}\right)\right) & =\left|\lambda-\lambda^{\prime}\right|-R_{\lambda}^{k}-2^{-3 k-3} \varepsilon e^{-C_{k+1} p\left(\lambda^{\prime}\right)-B_{k+1}} \\
& \geq 2^{-3 k-2} \varepsilon e^{-C_{k} p(\lambda)-B_{k}}-2^{-3 k-3} \varepsilon e^{-C_{k} p(\lambda)-B_{k}} \\
& \geq 2^{-3 k-3} \varepsilon e^{-C p(\lambda)} .
\end{aligned}
$$

\section{Proof of Theorem 1.4. Necessity}

Assume $\mathcal{R}_{\Lambda}\left(A_{p}\right)=X_{p}^{n-1}(\Lambda), n \geq 2$. Using Proposition 2.1, we have $X_{p}^{n-1}(V)=X_{p}^{n}(V)$, and by Lemma 2.4 we deduce that $\Lambda=\Lambda_{1} \cup \cdots \cup \Lambda_{n}$, where $\Lambda_{1}, \ldots, \Lambda_{n}$ are weakly separated sequences. We want to show that each $\Lambda_{j}$ is an interpolating sequence.

Let $\omega\left(\Lambda_{j}\right) \in A_{p}\left(\Lambda_{j}\right)=X_{p}^{0}\left(\Lambda_{j}\right)$. Let $\cup_{\lambda \in \mathcal{L}} D_{\lambda}$ be the covering of $\Lambda$ given by Lemma 2.6. We extend $\omega\left(\Lambda_{j}\right)$ to a sequence $\omega(\Lambda)$ which is constant on each $D_{\lambda} \cap \Lambda_{j}$ in the following way:

$$
\omega_{\mid D_{\lambda} \cap \Lambda}= \begin{cases}0 & \text { if } D_{\lambda} \cap \Lambda_{j}=\emptyset \\ \omega(\alpha) & \text { if } D_{\lambda} \cap \Lambda_{j}=\{\alpha\} .\end{cases}
$$


We verify by induction that the extended sequence is in $X_{p}^{k-1}(\Lambda)$ for all $k$. It is clear that it belongs to $X_{p}^{0}(\Lambda)$. Assume that $\omega \in X_{p}^{k-2}(\Lambda)$ and consider $\left(\alpha_{1}, \ldots, \alpha_{k}\right) \in \Lambda^{k}$. If all the points are in the same $D_{\lambda}$ then $\Delta^{k-1} \omega\left(\alpha_{1}, \ldots, \alpha_{k}\right)=0$, so we may assume that $\alpha_{1} \in D_{\lambda}$ and $\alpha_{k} \in D_{\lambda^{\prime}}$ with $\lambda \neq \lambda^{\prime}$. Then we have

$$
\left|\alpha_{1}-\alpha_{k}\right| \geq a \varepsilon e^{-B_{1} p(\lambda)},
$$

by Lemma 2.6 (iii). With this and the induction hypothesis it is clear that for certain constants $A, B>0$

$$
\begin{aligned}
\left|\Delta^{k-1} \omega\left(\alpha_{1}, \ldots, \alpha_{k}\right)\right| & =\left|\frac{\Delta^{k-2} \omega\left(\alpha_{2}, \ldots, \alpha_{k}\right)-\Delta^{k-2} \omega\left(\alpha_{1}, \ldots, \alpha_{k}\right)}{\alpha_{1}-\alpha_{k}}\right| \\
& \leq A e^{B\left[p\left(\alpha_{1}\right)+\cdots+p\left(\alpha_{k}\right)\right]} .
\end{aligned}
$$

In particular $\omega(\Lambda) \in X_{p}^{n-1}(\Lambda)$, and by assumption, there exist $f \in A_{p}$ interpolating the values $\omega(\Lambda)$. In particular $f$ interpolates $\omega\left(\Lambda_{j}\right)$.

\section{Proof of Theorem 1.4. Sufficiency}

According to Proposition 2.1 we only need to see that $X_{p}^{n-1}(\Lambda) \subset$ $\mathcal{R}_{\Lambda}\left(A_{p}\right)$.

Before going further, let us recall the following facts about interpolation in the spaces $A_{p}$.

Lemma 4.1. [1, Lemma 2.2.6] Let $\Gamma$ be an $A_{p}$-interpolating sequence. Then:

(i) For all $A, B>0$, there exist constants $A^{\prime}, B^{\prime}>0$ such that for all sequences $\omega \in A_{p}(\Gamma)$ with $\sup _{\gamma \in \Gamma}|\omega(\gamma)| e^{-B p(\gamma)} \leq A$ there exists $f \in A_{p}$ with $\sup _{z}|f(z)| e^{-B^{\prime} p(z)} \leq A^{\prime}$ and $f(\gamma)=\omega(\gamma)$ for all $\gamma \in \Gamma$.

(ii) There exists a constant $C>0$ such that $\sum_{\gamma \in \Gamma} e^{-C p(\gamma)}<\infty$.

Applying (i) to the sequences $\omega_{\gamma}=\left\{\delta_{\gamma, \gamma^{\prime}}\right\}_{\gamma^{\prime} \in \Gamma}$ it is easy to deduce that $\Gamma$ is weakly separated. Property (ii) is just a consequence of the weak separation and properties (w1) and (w2).

Assume thus that $\Lambda=\Lambda_{1} \cup \cdots \cup \Lambda_{n}$ where $\Lambda_{1}, \ldots, \Lambda_{n}$ are interpolating sequences. Recall that each $\Lambda_{j}$ is weakly separated (Corollary 2.5). Consider also the covering of $\Lambda$ given by Lemma 2.6. 
Lemma 4.2. There exist constants $A, B>0$ and a sequence $\left\{F_{\lambda}\right\}_{\lambda \in \mathcal{L}} \subset$ $A_{p}$ such that:

$$
\begin{aligned}
& F_{\lambda}(\alpha)= \begin{cases}1 & \text { if } \alpha \in \Lambda \cap D_{\lambda} \\
0 & \text { if } \alpha \in \Lambda \cap D_{\lambda^{\prime}}, \lambda^{\prime} \neq \lambda\end{cases} \\
& \left|F_{\lambda}(z)\right| \leq A e^{B(p(\lambda)+p(z))} \text { for all } z \in \mathbb{C} .
\end{aligned}
$$

Proof. Fix $\lambda \in \mathcal{L}$ and define $\omega(\Lambda)$ by

$$
\omega(\alpha)= \begin{cases}\prod_{\beta \in \Lambda \cap D_{\lambda}}(\alpha-\beta)^{-1} & \text { if } \alpha \notin \Lambda \cap D_{\lambda} \\ 0 & \text { if } \alpha \in \Lambda \cap D_{\lambda} .\end{cases}
$$

By Lemma 2.6 (iii), we have $|\alpha-\beta| \geq c \varepsilon e^{-C p(\alpha)}$ whenever $\alpha \notin \Lambda \cap D_{\lambda}$, $\beta \in \Lambda \cap D_{\lambda}$. Since $\#\left(\Lambda \cap D_{\lambda}\right) \leq n$ we deduce that

$$
|\omega(\alpha)| \leq(c \varepsilon)^{-n} e^{n C p(\alpha)}
$$

Recall that $\Lambda_{j}$ is an interpolating sequence for all $j=1, \ldots, n$, thus there exist a $n$-indexed sequence $\left\{f_{\lambda, j}\right\}_{\lambda \in \mathcal{L}, j \in[1, n]]} \subset A_{p}$ such that for all $z \in \mathbb{C}$,

$$
\begin{aligned}
\left|f_{\lambda, j}(z)\right| & \leq A e^{B p(z)} \\
f_{\lambda, j}(\alpha) & =\prod_{\beta \in \Lambda \cap D_{\lambda}}(\alpha-\beta)^{-1} \text { if } \alpha \notin \Lambda_{j} \cap D_{\lambda},
\end{aligned}
$$

with the constants $A$ and $B$ independent of $\lambda$ (see Lemma 4.1(i)).

The sequence of functions $\left\{F_{\lambda}\right\}_{\lambda \in \mathcal{L}}$ defined by

$$
F_{\lambda}(z)=\prod_{j=1}^{n}\left[1-\prod_{\beta \in \Lambda \cap D_{\lambda}}(z-\beta) f_{\lambda, j}(z)\right]
$$

has the desired properties.

Lemma 4.3. For all $D>0$, there exist $D^{\prime}>0$ and a sequence $\left\{G_{\lambda}\right\}_{\lambda \in \mathcal{L}} \subset A_{p}$ such that:

$$
\begin{aligned}
& G_{\lambda}(\alpha)=e^{D p(\lambda)} \quad \text { if } \quad \alpha \in \Lambda \cap D_{\lambda} . \\
& \left|G_{\lambda}(z)\right| \leq A e^{B p(\lambda)} e^{D^{\prime} p(z)} \quad \text { for all } z \in \mathbb{C},
\end{aligned}
$$

where $A, B>0$ do not depend on $D$.

Proof. In this proof $D^{\prime}$ denotes a constant depending on $D$ but not on $\lambda$, and its actual value may change from one occurrence to the other. 
Let $\lambda \in \mathcal{L}$. Assume, without loss of generality, that $D_{\lambda} \cap \Lambda_{j}=\left\{\alpha_{\lambda, j}\right\}$ for all $j$. As $\Lambda_{1}$ is an interpolating sequence and $e^{D p(\lambda)} \leq A e^{D^{\prime} p\left(\alpha_{\lambda, 1}\right)}$, by Lemma 4.1 (i) there exists a sequence $\left\{h_{\lambda, 1}\right\}_{\lambda} \subset A_{p}$ such that

$$
\begin{aligned}
& h_{\lambda, 1}\left(\alpha_{\lambda, 1}\right)=e^{D p(\lambda)} \\
& \left|h_{\lambda, 1}(z)\right| \leq A e^{D^{\prime} p(z)} \quad \text { for all } z \in \mathbb{C} .
\end{aligned}
$$

Setting $H_{\lambda, 1}(z)=h_{\lambda, 1}(z)$, we have $H_{\lambda, 1}\left(\alpha_{\lambda, 1}\right)=e^{D p(\lambda)}$. Now, as $\Lambda_{2}$ is $A_{p}$-interpolating and

$$
\frac{\left|e^{D p(\lambda)}-H_{\lambda, 1}\left(\alpha_{\lambda, 2}\right)\right|}{\left|\alpha_{\lambda, 2}-\alpha_{\lambda, 1}\right|}=\frac{\left|H_{\lambda, 1}\left(\alpha_{\lambda, 1}\right)-H_{\lambda, 1}\left(\alpha_{\lambda, 2}\right)\right|}{\left|\alpha_{\lambda, 2}-\alpha_{\lambda, 1}\right|} \leq A e^{D^{\prime} p\left(\alpha_{\lambda, 2}\right)},
$$

there exists a sequence $\left\{h_{\lambda, 2}\right\}_{\lambda} \subset A_{p}$ such that

$$
\begin{aligned}
& h_{\lambda, 2}\left(\alpha_{\lambda, 2}\right)=\frac{e^{D p(\lambda)}-H_{\lambda, 1}\left(\alpha_{\lambda, 2}\right)}{\alpha_{\lambda, 2}-\alpha_{\lambda, 1}} \\
& \left|h_{\lambda, 2}(z)\right| \leq A e^{D^{\prime} p(z)} \text { for all } z \in \mathbb{C} .
\end{aligned}
$$

Setting $H_{\lambda, 2}(z)=h_{\lambda, 1}(z)+h_{\lambda, 2}(z)\left(z-\alpha_{\lambda, 1}\right)$. We have

$$
H_{\lambda, 2}\left(\alpha_{\lambda, 1}\right)=H_{\lambda, 2}\left(\alpha_{\lambda, 2}\right)=e^{D p(\lambda)} .
$$

We proceed by induction to construct a sequence of functions $\left\{h_{\lambda, k}\right\}_{\lambda} \subset$ $A_{p}$ such that

$$
\begin{aligned}
& h_{\lambda, k}\left(\alpha_{\lambda, k}\right)=\frac{e^{D p(\lambda)}-H_{\lambda, k-1}\left(\alpha_{\lambda, k}\right)}{\left(\alpha_{\lambda, k}-\alpha_{\lambda, 1}\right) \cdots\left(\alpha_{\lambda, k}-\alpha_{\lambda, k-1}\right)} \\
& \left|h_{\lambda, k}(z)\right| \leq A e^{D^{\prime} p(z)} \text { for all } z \in \mathbb{C} .
\end{aligned}
$$

Then the function defined by $H_{\lambda, k}(z)=H_{\lambda, k-1}(z)+h_{\lambda, k}(z)(z-$ $\left.\alpha_{\lambda, 1}\right) \cdots\left(z-\alpha_{\lambda, k-1}\right)$ verifies

$$
H_{\lambda, k}\left(\alpha_{\lambda, 1}\right)=\cdots=H_{\lambda, k}\left(\alpha_{\lambda, k}\right)=e^{D p(\lambda)} .
$$

Finally, we set $G_{\lambda}=H_{\lambda, n}$.

To proceed with the proof of the inclusion $X_{p}^{n-1}(\Lambda) \subset \mathcal{R}_{\Lambda}\left(A_{p}\right)$, let $\omega(\Lambda) \in X_{p}^{n-1}(\Lambda)$.

Fix $\lambda \in \mathcal{L}$ and let $\Lambda \cap D_{\lambda}=\left\{\alpha_{1}, \ldots, \alpha_{k}\right\}, k \leq n$. We first consider a polynomial interpolating the values $\omega\left(\alpha_{1}\right), \ldots, \omega\left(\alpha_{k}\right)$ :

$$
P_{\lambda}(z)=\Delta^{0} \omega\left(\alpha_{1}\right)+\Delta^{1} \omega\left(\alpha_{1}, \alpha_{2}\right)\left(z-\alpha_{1}\right)+\cdots+\Delta^{k-1} \omega\left(\alpha_{1}, \ldots, \alpha_{k}\right) \prod_{j=1}^{k-1}\left(z-\alpha_{j}\right) .
$$


Notice that $P_{\lambda} \in A_{p}$, since $\omega(\Lambda) \in X_{p}^{n-1}(\Lambda)$ and by properties (w1) and (w2) we have

$$
\left|P_{\lambda}(z)\right| \leq A|z|^{k} e^{B\left[p\left(\alpha_{1}\right)+\cdots+p\left(\alpha_{k}\right)\right]} \leq A e^{B^{\prime}[p(z)+p(\lambda)]} .
$$

Now, define

$$
f=\sum_{\lambda \in \mathcal{L}} F_{\lambda} G_{\lambda} P_{\lambda} e^{-D p(\lambda)},
$$

where $D$ is a large constant to be chosen later on.

By the preceding estimates on $G_{\lambda}$ and $P_{\lambda}$, there exist constants $A, B>0$ not depending on $D$ and a constant $D^{\prime \prime}>0$ such that, for all $z \in \mathbb{C}$, we have

$$
|f(z)| \leq A e^{D^{\prime \prime} p(z)} \sum_{\lambda \in \mathcal{L}} e^{(B-D) p(\lambda)} .
$$

In view of Lemma 4.1(ii), taking $D=B+C$, the latter sum converges and $f \in A_{p}$.

To verify that $f$ interpolates $\omega(\Lambda)$, let $\alpha \in \Lambda$ and let $\lambda$ be the (unique) point of $\mathcal{L}$ such that $\alpha \in D_{\lambda}$. Then, $f(\alpha)=G_{\lambda}(\alpha) P_{\lambda}(\alpha) e^{-D p(\alpha)}=$ $P_{\lambda}(\alpha)=\omega(\alpha)$, as desired.

\section{Similar RESUlts in the Disk}

The previous definitions and proofs can be adapted to produce analogous results in the disk. To do so one just needs to replace the Euclidean distance used in $\mathbb{C}$ by the pseudo-hyperbolic distance

$$
\rho(z, \zeta)=\left|\frac{z-\zeta}{1-\bar{\zeta} z}\right| \quad z, \zeta \in \mathbb{D},
$$

and the Euclidean divided differences by their hyperbolic version

$$
\begin{aligned}
\delta^{0} \omega\left(\lambda_{1}\right) & =\omega\left(\lambda_{1}\right), \\
\delta^{j} \omega\left(\lambda_{1}, \ldots, \lambda_{j+1}\right) & =\frac{\Delta^{j-1} \omega\left(\lambda_{2}, \ldots, \lambda_{j+1}\right)-\Delta^{j-1} \omega\left(\lambda_{1}, \ldots, \lambda_{j}\right)}{\frac{\lambda_{j+1}-\lambda_{1}}{1-\lambda_{1} \lambda_{j+1}}} \quad j \geq 1 .
\end{aligned}
$$

In this context a function $\phi: \mathbb{D} \longrightarrow \mathbb{R}_{+}$is a weight if

(wd1) There is a constant $K>0$ such that $\phi(z) \geq K \ln \left(\frac{1}{1-|z|}\right)$.

$\left(\right.$ wd2) There are constants $D_{0}>0$ and $E_{0}>0$ such that whenever $\rho(z, \zeta) \leq 1$ then

$$
\phi(z) \leq D_{0} \phi(\zeta)+E_{0}
$$

The model for the associated spaces

$$
A_{\phi}=\left\{f \in H(\mathbb{D}): \sup _{z \in \mathbb{D}}|f(z)| e^{-B \phi(z)}<\infty \text { for some } B>0\right\},
$$


is the Korenblum algebra $A^{-\infty}$, which corresponds to the choice $e^{-\phi(z)}=$ $1-|z|$. The interpolating sequences for this and similar algebras have been characterised in [3] and [4].

With these elements, and replacing the factors $z-\alpha$ by $\frac{z-\alpha}{1-\bar{\alpha} z}$ when necessary, we can follow the proofs above and, mutatis mutandis, show that Theorem 1.4 also holds in this situation.

The only point that requires further justification is the validity of Lemma 4.1 for the weights $\phi$. Condition (i) is a standard consequence of the open mapping theorem for ( $\mathrm{LF}$ )-spaces applied to the restriction map $\mathcal{R}_{\Lambda}$, and the same proof as in [1, Lemma 2.2.6] holds. Applying (i) to the sequences $\omega_{\lambda}(\Lambda)$ defined by

$$
\omega_{\lambda}\left(\lambda^{\prime}\right)= \begin{cases}1 & \text { if } \lambda^{\prime}=\lambda \\ 0 & \text { if } \lambda^{\prime} \neq \lambda\end{cases}
$$

we have functions $f_{\lambda} \in A_{\phi}$ interpolating these values and with growth control independent of $\lambda$. Since $1=\left|f_{\lambda}(\lambda)-f_{\lambda}\left(\lambda^{\prime}\right)\right|$, an estimate on the derivative of $f_{\lambda}$ shows that for some $C>0$ and $\varepsilon>0$ the pseudohyperbolic disks $D_{H}\left(\lambda, \varepsilon e^{-C \phi(\lambda)}\right)=\left\{z \in \mathbb{D}: \rho(z, \lambda)<e^{-C \phi(\lambda)}\right\}$ are pairwise disjoint. In particular the sum of their areas is finite, hence

$$
\sum_{\lambda \in \Lambda}(1-|\lambda|)^{2} e^{-2 C \phi(\lambda)}<+\infty
$$

From this and condition (wd1) we finally obtain (ii).

\section{REFERENCES}

[1] Berenstein, C.A. - Gay, R. Complex Analysis and Special Topics in Harmonic Analysis, Springer Verlag, 1995.

[2] C.A. Berenstein and B.Q. Li, Interpolating varieties for spaces of meromorphic functions, J. Geom. Anal. 5 (1995), 1-48.

[3] J. Bruna and D. Pascuas, Interpolation in $A^{-\infty}$, J. London Math. Soc. (2) 40 (1989), no. 3, 452-466.

[4] X. Massaneda, Density conditions for interpolation in $A^{-\infty}$, J. Anal. Math. 79 (1999), 299-314.

[5] X. Massaneda, J. Ortega-Cerdà and M. Ounaïes. A geometric characterization of interpolation in $\hat{\mathcal{E}}^{\prime}(\mathbb{R})$, Trans. Amer. Math. Soc. 358 (2006), 3459-3472.

[6] M. Ounaïes. Interpolation by entire functions with growth conditions. To appear in Mich. Math. J.

[7] Vasyunin, V. I. Traces of bounded analytic functions on finite unions of Carleson sets (Russian). Investigations on linear operators and the theory of functions, XII. Zap. Nauchn. Sem. Leningrad. Otdel. Mat. Inst. Steklov. (LOMI) 126 (1983), 31-34. 
[8] Vasyunin, V. I. Characterization of finite unions of Carleson sets in terms of solvability of interpolation problems (Russian). Investigations on linear operators and the theory of functions, XIII. Zap. Nauchn. Sem. Leningrad. Otdel. Mat. Inst. Steklov. (LOMI) 135 (1984), 31-35.

Departament de Matemàtica Aplicada i Anàlisi, Universitat de BarCelona, Gran Via 585, 08071-Barcelona, Spain

E-mail address: xavier.massaneda@ub.edu

Departament de Matemàtica Aplicada i Anàlisi, Universitat de BarCElona, Gran Via 585, 08071-Barcelona, Spain

E-mail address: jortega@ub.edu

Institut de Recherche Mathématique Avancée, Université Louis Pasteur, 7 Rue René Descartes, 67084 Strasbourg CEDEX, France

E-mail address: ounaies@math.u-strasbg.fr 